\title{
Adormecer a Ver Televisão como Fator de Risco para Perturbação do Sono na Idade Escolar
}

\section{Falling Asleep Watching Television as a Risk Factor for Sleep Disturbance in School Age}

Sandra Afonso ${ }^{1}$, Filipe Glória e Silva ${ }^{1}$, Cláudia Rocha Silva ${ }^{1}$, Ana Serrão Neto ${ }^{1}$

\section{RESUMO}

INTRODUÇÃO: O objetivo deste estudo foi avaliar a relação do hábito de adormecer a ver televisão com as perturbações do sono na idade escolar.

MATERIAL E MÉTODOS: Estudo transversal envolvendo turmas de primeiro ciclo de 17 agrupamentos escolares das áreas da Grande Lisboa, Península de Setúbal e Alentejo. Foi utilizada a versão portuguesa do Children's Sleep Habits Questionnaire (CSHQ-PT).

RESULTADOS: Foram considerados questionários de 996 crianças com idades entre os 6 e os 10 anos. Adormecer a ver televisão ocorria habitualmente em 167 crianças (16,8\%), às vezes em 294 crianças (29,5\%) e raramente em 535 crianças (53,7\%). Comparando as crianças dos subgrupos extremos (habitualmente e raramente, $n=702$ ), as crianças com o hábito de adormecer a ver televisão mostraram mais problemas do sono $(p<0,001)$ com cotações superiores nas seguintes escalas: Índice de Perturbação do Sono, Resistência em Ir para a Cama, Duração do Sono, Ansiedade Associada o Sono, Despertares Noturnos, Parassónias e Sonolência Diurna. Verificou-se uma correlação positiva do hábito de adormecer a ver televisão com o Índice de Perturbação do Sono $(0,27)$ e uma correlação negativa com a escolaridade dos pais $(-0,22)$.

CONCLUSÃO: O hábito de adormecer a ver televisão pode ser encarado como um fator de risco para perturbação do sono nas crianças, devendo este tema ser abordado nas consultas de vigilância de saúde da criança, numa perspetiva preventiva deste tipo de perturbação.

PALAVRAS-CHAVE: Criança; Distúrbios da Iniciação e Manutenção do Sono; Inquéritos e Questionários; Sono; Televisão 


\section{ABSTRACT \\ INTRODUCTION: The aim of this study was to evaluate the relation between falling asleep watching television and sleep dis- turbances in school age children.}

MATERIAL AND METHODS: Cross-sectional study, involving 17 elementary schools in Lisbon, Setúbal Peninsula and Alentejo. The sleep habits were evaluated with the Portuguese version of the Children's Sleep Habits Questionnaire (CSHQ-PT).

RESULTS: A total of 996 questionnaires were analyzed, from children aged 6 to 10 years. Falling asleep watching television occurred usually in 167 children (16.8\%), sometimes in 294 children (29.5\%) and rarely in 535 children (53.7\%). Comparing children in extreme subgroups (usually and rarely, $n=702$ ), children with the habit of falling asleep watching television developed more sleep problems with higher odds on the following scales ( $p$ 0.001): Sleep disturbance Index, Bedtime Resistance, Sleep Duration, Sleep Anxiety, Night Awakenings, Parasomnias and Daytime Sleepiness. There was a positive correlation between the habit of falling asleep watching television with the Sleep Disorder Index (0.27) and inverse with parents' education (-0.22).

CONCLUSION: The habit of falling asleep watching television can be seen as a risk factor for sleep disturbance, and this topic should be addressed in the child health surveillance appointments as primary prevention.

KEYWORDS: Child; Sleep; Sleep Initiation and Maintenance Disorders; Surveys and Questionnaires; Television

\section{INTRODUÇÃO}

O sono é considerado particularmente importante para a aprendizagem e memória, tendo também implicações na regulação emocional e do comportamento, ${ }^{1}$ assim como a nível cardiovascular, imunológico e metabólico, ${ }^{2}$ está particularmente associado ao risco de obesidade. $^{3}$ Os pais nem sempre valorizam as questões do sono ou reconhecem determinados comportamentos como problemáticos, uma vez que têm hábitos, padrões e expectativas próprios, de acordo com as suas normas culturais, étnicas e nível socioeconómico. ${ }^{2}$ A prevalência das perturbações do sono nas crianças é variável, com uma estimativa de 20\% - 30\%, predominando na idade pré-escolar. ${ }^{4}$ Em Portugal, numa amostra comunitária de 966 crianças dos 2 aos 10 anos, o rastreio das perturbações com questionário foi positivo em mais de metade das crianças (74\%), ${ }^{4}$ enquanto noutro estudo, a maioria das crianças em idade pré-escolar foi classificada como tendo uma boa qualidade de sono, na perceção dos pais. ${ }^{5}$

Na era da tecnologia, os ecrãs têm um papel central na vida da maior parte das crianças. Nos EUA, 97\% dos adolescentes têm pelo menos um dispositivo eletrónico no quarto, ${ }^{1}$ sendo a presença da televisão (TV) mais prevalente do que na Europa. ${ }^{6} \mathrm{O}$ uso da TV como rotina de sono antes de ir para a cama é uma prática de muitas famílias, globalmente generalizada, com trabalhos que apontam entre os 53\% e 60\% em crianças de idade pré-escolar na Tailândia e Chile 7,8 respetivamente, assim como 76,5\% em crianças dos 4 aos 10 anos nos EUA. . $^{1,9}$

Vários mecanismos têm sido propostos para explicar a interferência da TV no sono. Pode existir uma alteração na arquitetura do sono, com diminuição de ondas lentas, sono REM e eficiência do sono. ${ }^{6}$ A luz do ecrã da TV causa uma supressão da secreção da melatonina (particularmente nas crianças, pelo maior diâmetro da pupila), interferindo no ritmo circadiano natural do sono.,10 A visualização de TV, particularmente determinados conteúdos, provoca uma hiperestimulação no cérebro das crianças, com excitação emocional, atrasando o início do sono. ${ }^{5,7,11}$

Existem vários trabalhos internacionais que relacionam a visualização de TV e presença desta no quarto de crianças com vários problemas e perturbações do sono, ${ }^{7}$ nomeadamente menor duração do sono e atraso da hora de deitar,1,3,6,7,11 maior resistência em ir para a cama, ${ }^{1}$ pior qualidade do sono, ${ }^{8,11}$ cansaço matinal e maior frequência de pesadelos, terrores noturnos e sonambulismo. ${ }^{7}$ Estas alterações não são transitórias, mantêm-se no tempo, tal como comprovado num estudo finlandês em crianças de 10 e 11 anos de idade, em que as alterações iniciais do sono foram identificadas dezoito meses depois. ${ }^{6}$ Dados da National Sleep Foundation (EUA) revelam que crianças dos 6 aos 10 anos de idade com TV no quarto dormem menos 9 minutos por dia, ${ }^{12}$ sendo reportado num estudo em Massachusetts, EUA, com 2048 estudantes dos 8 aos 12 anos, uma diminuição de 18 minutos, independentemente do uso ou presença concomitante no quarto, dos chamados pequenos ecrãs. ${ }^{3,5}$ Num estudo longitudinal, com crianças dos 6 meses aos 7 anos de idade, verificou-se uma maior vulnerabilidade aos efeitos negativos da TV sobre o sono no sexo masculino ${ }^{12,13}$ e minorias raciais / étnicas, ${ }^{13}$ atribuída a suscetibilidade diferente, de género e raça, aos estímulos.

A nível nacional, nos últimos anos, surgiram alguns estudos de caracterização dos hábitos de sono da popula- 
ção pediátrica portuguesa, tanto em idade escolar como pré-escolar. Há consenso sobre a existência de problemas do sono, nomeadamente duração total do sono inferior às recomendações internacionais para a faixa etária, ${ }^{4-5,14-16}$ assim como sobre os índices particulares ao nível da qualidade do sono, ${ }^{4,17}$ hora de deitar, ${ }^{15}$ resistência em ir para a cama, despertares noturnos e parassónias. ${ }^{14,17} \mathrm{O}$ estudo "Hábitos e problemas do sono dos dois aos dez anos", com uma amostra de 1450 crianças de várias zonas do país, reportou que o hábito de adormecer a ver TV era frequente, sendo habitual em 12\% das crianças dos 2 aos 3 anos, 15\% dos 4 aos 5 anos e $17 \%$ dos 6 aos 10 anos. ${ }^{18}$

O presente estudo tem como objetivo, a partir da amostra anterior, mas na faixa etária dos 6 aos 10 anos, avaliar a relação do hábito de adormecer a ver TV com as perturbações do sono, prevendo-se uma associação positiva com implicações no âmbito clínico.

\section{MÉTODOS}

Foi realizado um estudo transversal abrangendo as unidades territoriais portuguesas da Grande Lisboa, Península de Setúbal e Alentejo onde residiam 34\% das crianças portuguesas à data dos Censos de 2011 do Instituto Nacional de Estatística. A amostra do estudo "Hábitos e problemas do sono dos dois aos dez anos" foi constituída por etapas com seleção de escolas de 17 agrupamentos escolares das unidades territoriais selecionadas como descrito previamente. ${ }^{14}$ Estes agrupamentos foram considerados por áreas geográficas de forma proporcional à população residente, quinze de forma aleatória e dois por conveniência (que entraram no estudo prévio de validação do questionário). Dentro de cada agrupamento, a amostra foi estratificada por anos escolares, sendo incluídas todas as crianças de uma turma.

O critério de inclusão na recolha de dados iniciais foi a idade da criança entre os 2 e os 10 anos sendo solicitado consentimento informado aos pais. Na presente análise, foi considerada apenas faixa etária dos 6 aos 10 anos. Os hábitos e problemas do sono das crianças foram avaliados através da versão portuguesa do Children's Sleep Habits Questionnaire (CSHQ-PT), previamente validado.18 Este questionário é preenchido pelos pais com base no comportamento e no padrão de sono da última semana. Relativamente à estrutura do questionário, a frequência de comportamentos-problema que constituem sintomas de distúrbios do sono é avaliada numa escala de três opções: "Acontece habitualmente" (cinco a sete vezes/semana), "Acontece às vezes" (duas a quatro vezes/semana) ou "Acontece raramente" (uma vez/semana ou nunca). As respostas são pontuadas de um a três de forma a que uma pontuação mais elevada corresponda a uma maior frequência de comportamentos-problema mais frequentes, ou seja, maior perturbação do sono. A escala de 33 itens está organizada em 8 subescalas de problemas do sono, cujos itens estão descritos na Fig. 1. A cotação total (IPS - Índice de Perturbação do Sono) e das subescalas é obtido pela soma das pontuações dos itens individuais. Foi considerado como ponto de corte do IPS para o rastreio das perturbações do sono um valor igual ou superior a $48 \mathrm{com}$ base num estudo prévio de validação com amostras clínicas de crianças com distúrbios do sono. ${ }^{20}$

O hábito de adormecer a ver TV foi avaliado através do respetivo item do questionário: "Adormece a ver televisão", com a mesma escala de resposta de três opções. Os pais indicaram também as horas de deitar e acordar em dias de semana e no fim-de-semana e a sua estimativa do tempo total de sono diário (TSD), referente a um dia típico de semana.

O questionário do estudo incluiu ainda questões adicionais ao CSHQ-PT inquirindo sobre dados sociodemográficos, tempo total diário de TV e videojogos, tempo diário de atividade desportiva, problemas de saúde e medicação habitual da criança. O nível educacional dos pais foi classificado em quatro classes, considerando a escolaridade mais elevada obtida: I - Inferior ao $3^{\circ}$ Ciclo; II - $3^{\circ}$ Ciclo; III - Ensino Secundário; IV - Bacharelato ou superior. Foi distribuído aos pais através do professor titular de cada turma, sendo entregue diretamente aos pais ou ao aluno.

Este estudo foi aprovado por Comissão de Ética, pelo Ministério da Educação e pela Comissão Nacional de Proteção de Dados Pessoais. Os questionários foram distribuídos entre janeiro e junho de 2011. A análise estatística dos dados foi realizada através do programa Statistical Package for the Social Sciences (SPSS), versão 11 (2001).

\section{RESULTADOS}

No estudo "Hábitos e problemas do sono dos dois aos dez" foram distribuídos 2257 questionários sendo devolvidos 1559 (69,1\%). Foram excluídos 109 por preenchimento inválido ou muito incompleto (mais de 20\% de respostas omissas). Considerando a faixa etária dos 6 aos 10 anos e os questionários com resposta ao item "adormece a ver TV" ( $n=996)$, este comportamento ocorria habitualmente em 167 crianças (16,8\%), às vezes em 294 crianças (29,5\%) e raramente em 535 crianças (53,7\%). Na análise subsequente, foram considerados 
Subescala 1. Resistência em ir para a cama

Item 1 Deita-se sempre à mesma hora

Item 3 Adormece sozinho na sua própria cama

Item 4 Adormece na cama dos pais ou dos irmãos

Item 5 Precisa dos pais no quarto para adormecer

Item 6 "Luta" na hora de deitar

Item 8 Tem medo de dormir sozinho

Subescala 2. Início do sono

Item 2 Demora até 20 minutos a adormecer

Subescala 3. Duração do sono

Item 9 Dorme pouco

Item 10 Dorme o que é necessário

Item 11 Dorme o mesmo número de horas todos os dias

Subescala 4. Ansiedade associada ao sono Item 5 Precisa dos pais no quarto para adormecer Item 7 Tem medo de dormir no escuro

Item 8 Tem medo de dormir sozinho

Item 21 Tem dificuldade em dormir fora de casa

Subescala 5. Despertares noturnos

Item 16 Vai para a cama dos pais, irmãos

Item 24 Acorda uma vez durante noite

Item 25 Acorda mais de uma vez durante a noite

Subescala 6. Parassónias

Item 12 Molha a cama à noite

Item 13 Fala a dormir

Item 14 Tem sono agitado, mexe-se muito a dormir Item 15 Anda a dormir, à noite (sonambulismo)

Item 17 Range os dentes durante o sono

Item 22 Acorda a gritar, a suar, inconsolável

Item 23 Acorda assustado com pesadelos

Subescala 7. Perturbação respiratória do sono Item 18 Ressona alto

Item 19 Parece parar de respirar durante o sono

Item 20 Ronca ou tem dificuldade em respirar durante o sono

Subescala 8. Sonolência diurna

Item 26 De manhã, acorda por si próprio

Item 2 Acorda mal-humorado

Item 28 É acordado pelos pais ou irmãos

Item 29 Tem dificuldade em sair da cama de manhã

Item 30 Demora a ficar bem acordado

Item 31 Parece cansado

Item 32 Sonolento/adormece a ver televisão

Item 33 Sonolento/adormece a andar de carro

Item extra na versão portuguesa

Item 34 Adormece a ver televisão

FIGURA 1. Subescalas do questionário (CSHQ-PT). apenas os grupos extremos com base nesta resposta (adormece a ver TV "habitualmente" versus "raramente", n= 702 crianças). A avaliação sociodemográfica desta amostra é apresentada na Tabela 1.

Não existiam diferenças significativas na distribuição de idades e sexo dos dois grupos de crianças que adormeciam com ou sem TV. O nível educacional dos pais tende a ser mais elevado no grupo que raramente adormece a ver TV. Não foram encontradas diferenças significativas nos dois grupos em relação à indicação de problemas de saúde $\left(\chi^{2}=0,84, p=0,66\right)$, particularmente perturbação do neurodesenvolvimento $\left(\chi^{2}=0,000285, p=0,99\right)$.

Sobre os horários, não se verificaram diferenças significativas nas horas de deitar e acordar nos dias de semana e no fim-de-semana. Em relação à duração do sono, o tempo total de sono diário estimado pelos pais foi inferior no grupo que adormece habitualmente a ver TV $(\bar{\chi}=9$ horas 18 minutos versus $\bar{\chi}=9$ horas 41 minutos nas crianças que raramente adormecem a ver TV, MW U= 23267, $p<0,001)$.

Os resultados do Índice de Perturbação do Sono, rastreio das perturbações do sono e cotações das subescalas do questionário, são apresentados na Tabela 2.

Assim, as crianças que adormecem habitualmente a ver TV mostraram valores mais elevados no Índice de Perturbação do Sono, no Rastreio de Perturbação do Sono e nas subescalas Resistência em Ir para a Cama, Duração do Sono (sono insuficiente e/ou com horário irregular), Ansiedade Associada ao Sono (dificuldade em adormecer sozinho), Despertares Noturnos e Sonolência Diurna.

Em relação ao tempo diário gasto a ver televisão e videojogos, este foi superior nas crianças que adormeciam habitualmente a ver TV $(\bar{\chi}=2$ horas 7 minutos versus $\bar{\chi}=1$ hora 48 minutos, $M N U=30134,5, p=0,002)$. No tempo de atividade desportiva durante a semana não foram encontradas diferenças significativas ( $p=0,61$, média global $\bar{\chi}=2$ horas 49 minutos).

\section{DISCUSSÃO}

De acordo com o nosso estudo, adormecer a ver TV é uma prática verificada em 46,3\% das crianças da amostra, um valor intermédio entre os 76\% referidos na literatura internacional, em 500 crianças americanas dos 4 aos 10 anos de idade ${ }^{9}$ e os 35,9\% descritos num estudo de crianças portuguesas, entre os 2 e 10 anos, na área do grande Porto. ${ }^{17}$ Esta diferença poderá estar relacionada com a maior abrangência dos grupos etários estudados, fatores socioeconómicos e culturais que influenciem o tipo de aparelhos eletrónicos usados e outras formas de adormecer. 
TABELA 1. Caracterização sociodemográfica dos grupos estudos.

\begin{tabular}{|c|c|c|c|}
\hline Variável & $\begin{array}{c}\text { Adormece } \\
\text { habitualmente a ver TV } \\
\mathrm{N}=167\end{array}$ & $\begin{array}{c}\text { Adormece } \\
\text { raramente a ver TV } \\
\mathrm{N}=535\end{array}$ & Testes estatísticos \\
\hline \multicolumn{4}{|l|}{ Idade } \\
\hline Média & 7,7 & 7,8 & \multirow{9}{*}{$\begin{array}{c}M N U=41947,5, p=0,22 \\
\text { T. E. Fischer } p=0,13 \\
\chi^{2}=40,55 p<0,001\end{array}$} \\
\hline Desvio-padrão & 1,3 & 1,3 & \\
\hline \multicolumn{4}{|l|}{ Sexo } \\
\hline Masculino & $73(43,7 \%)$ & $270(50,5 \%)$ & \\
\hline Feminino & $94(56,3 \%)$ & $265(49,5 \%)$ & \\
\hline \multicolumn{3}{|l|}{ Nível educacional dos pais } & \\
\hline I. Inferior ao $9^{\circ}$ ano & $37(22,2 \%)$ & $47(8,8 \%)$ & \\
\hline $11.9^{\circ}$ ano & $40(24,0 \%)$ & 107 (24,0\%) & \\
\hline II. $12^{\circ}$ ano & $61(36,5 \%)$ & $165(30,8 \%)$ & \\
\hline IV. Bacharelato/licenciat. & $29(17,4 \%)$ & $216(40,4 \%)$ & \\
\hline
\end{tabular}

Notas: $\mathrm{MN}=$ Teste de Mann-Whitney; $T$. E. Fischer = Teste exato de Fischer

TABELA 2. Resultados do questionário de sono.

\begin{tabular}{|c|c|c|c|}
\hline Variável & $\begin{array}{c}\text { Adormece } \\
\text { habitualmente a ver TV } \\
\mathrm{N}=167\end{array}$ & $\begin{array}{c}\text { Adormece } \\
\text { raramente a ver TV } \\
\mathrm{N}=535\end{array}$ & Testes estatísticos \\
\hline Índice de perturbação do sono & $49,6 \pm 8,0$ & $44,7 \pm 6,2$ & $M N U=19809, p<0,001$ \\
\hline $\begin{array}{l}\text { Rastreio de perturbação do sono positivo } \\
\text { (ponto de corte } 48 \text { ) }\end{array}$ & $56,9 \%$ & $28,1 \%$ & $\chi^{2}=37,42, p<0,001$ \\
\hline \multicolumn{4}{|l|}{ Cotação das subescalas } \\
\hline 1. Resistência ir p/ cama & 9,1 & 7,5 & $M N U=27,697, p<0,001$ \\
\hline 2. Início do sono & 1,9 & 1,9 & $M N U=43,401, p=0,63$ \\
\hline 3. Duração do sono & 4,2 & 3,8 & $M N U=32,739, p<0,001$ \\
\hline 4. Ansiedade no sono & 6,6 & 5,4 & $M N U=28,634, p<0,001$ \\
\hline 5. Despertares noturnos & 4,1 & 3,6 & $M N U=33,197, p<0,001$ \\
\hline 6. Parassónias & 9,4 & 8,8 & $M N U=32,621, p<0,001$ \\
\hline 7. Pert. resp. do sono & 3,7 & 3,5 & $M N U=39,654,5, p=0,10$ \\
\hline 8. Sonolência diurna & 14,1 & 13,0 & $M N U=32,520,5, p<0,001$ \\
\hline
\end{tabular}

Legenda: $M N$ = Teste de Mann-Whitney

É reconhecido o papel relevante dos aspetos culturais nos hábitos e política familiar do uso da televisão, inclusive na hora de dormir. ${ }^{13}$ A existência de múltiplos apareIhos de TV em casa, incluindo no quarto de dormir, facilita e promove o seu uso ao adormecer. ${ }^{1}$ Adormecer a ver TV é uma prática de facilitação parental, habitualmente como resposta à resistência em ir para a cama e demora a adormecer, mas funciona apenas como solução de curto prazo, uma vez que a criança se torna dependente deste estímulo. ${ }^{19,21}$

Dado que o comportamento de adormecer a ver TV mostrou associação com o nível educacional dos pais e com o índice de perturbação do sono (IPS), foram analisadas as correlações destas variáveis (correlações de Spearman por não serem cumpridos os pressupostos da correlação de Pearson). Verificou-se uma correlação significativa entre adormecer a ver TV e o IPS $\left(r_{s}=0,27, p\right.$ $<0,001)$, sendo menos frequente nas famílias com nível educacional mais elevado, como descrito noutro traba-
Iho.? As associações das outras variáveis também foram significativas: adormecer a ver TV e o nível educacional dos pais $\left(r_{s}=-0,22, p<0,001\right)$; bem como IPS e o nível educacional dos pais $\left(r_{s}=-0,16, p<0,001\right)$. Assim, o nível educacional apresentou uma relação inversa com o Índice de Perturbação do Sono, em consonância com a relação direta entre a escolaridade da mãe e o número de horas de sono, reportada anteriormente na população portuguesa. ${ }^{15}$ Neste grupo de crianças, a menor escolaridade da família parece associar-se a mais problemas comportamentais do sono, possivelmente influenciados pela subvalorização do tempo de sono necessário, diferenças nos estilos educativos ou menor valorização da importância do sono, o que leva ao não reconhecimento desta perturbação.

Embora estudos relatem nos rapazes um maior impacto do adormecer a ver TV na sonolência ${ }^{2}$ e no adormecer mais tarde, ${ }^{6}$ assim como descrevam o aumento da frequência das alterações no sono nas crianças mais velhas, 
presumivelmente pela maior exposição passiva à TV sem controlo parental ${ }^{7}$ e conteúdo mais violento dos programas visualizados, ${ }^{3}$ no nosso trabalho não existiram diferenças significativas em relação à idade e sexo das crianças nos grupos analisados.

Constatámos que o tempo diário gasto a ver televisão e videojogos foi significativamente superior nas crianças que adormecem habitualmente a ver TV. Este aspeto é relevante porque está descrito que mais de uma hora despendida em tais atividades aumenta significativamente o risco de sonolência diurna. ${ }^{2}$ Num grupo mais restrito de crianças portuguesas, entre os 3 e 6 anos de idade, o tempo diário gasto pelas crianças com o uso de pequenos ecrãs não foi considerado motivo de preocupação para os pais (menos de uma hora nos dias de semana, em 83,8\% das crianças), ${ }^{5}$ mas sendo este um hábito que se torna mais frequente com a idade, é importante a promoção do seu uso com limites bem definidos, desde a idade pré-escolar.

No nosso estudo, o hábito de adormecer a ver TV mostrou associação com problemas do sono, relacionados com a resistência em ir para a cama, duração do sono, ansiedade relacionada com o sono (dificuldade em adormecer sozinho), despertares noturnos, parassónias e sonolência diurna, dados semelhantes à literatura. ${ }^{1,3}$ De facto, estudos prévios mostraram que o hábito de ver TV ao adormecer constitui um forte fator preditivo para uma menor duração do sono ${ }^{9}$ e para o atraso do adormecer, ${ }^{6}$ em consonância com os nossos resultados.

Para além do efeito direto no sono, o adormecer a ver TV pode interferir também noutras atividades relacionadas com a higiene do sono, uma vez que habitualmente se relaciona com uma diminuição da atividade física ${ }^{5}$ e tendência para o sedentarismo, ${ }^{1}$ aumentando o risco de obesidade. No nosso estudo, contudo, não se encontraram diferenças significativas no tempo de atividade desportiva.

Apesar da maioria dos estudos apontar para uma duração total de sono insuficiente nas crianças, o reconhecimento dos pais sobre a inadequação do tempo de sono e distúrbios do sono nos seus filhos é baixa.,13,14 De igual modo, apenas 6,5\% dos pais revelaram consciência do efeito negativo da TV no sono das crianças.1,13 Estes itens não fazem parte do questionário usado no nosso estudo.

Os autores reconhecem outras limitações ao presente estudo. Apesar do período de recolha dos dados não ser atual, a análise e a relação entre as variáveis estudadas neste trabalho mantém o seu interesse, dada a sua pertinente importância na vigilância da saúde das crianças, não existindo muitos trabalhos publicados no contexto português, particularmente com este tamanho de amostra. Por outro lado, o foco deste trabalho foi o comportamento de adormecer a ver TV, não tendo sido questionados o uso de outros tipos de ecrãs, como o computador, telemóveis, tablets, que poderão substituir a TV com este fim. Também não pode ser descartado o enviesamento dos resultados pela chamada "resposta correta", que pode não corresponder totalmente à verdade, uma vez que os pais tinham conhecimento do objetivo do estudo. Seria interessante numa investigação futura, com um modelo de desenho longitudinal, avaliar o efeito dos vários tipos de dispositivos eletrónicos no sono, ao nível dos conteúdos visualizados, tempo de exposição, intervalo entre exposição e adormecer, assim como explorar o papel de fatores adicionais como os estilos parentais e o stress familiar.

Globalmente, consideramos que inquirir sobre este hábito no contexto clínico pode constituir uma forma de rastreio das perturbações do sono. Quando a criança apresenta este hábito, o sono deve ser avaliado de forma mais detalhada, abordando os problemas detetados. É também necessário entender o funcionamento e rotinas da família e as suas convicções sobre o sono, de forma a selecionar estratégias de intervenção eficazes. Perante a presença de problemas persistentes, particularmente com consequências diurnas, é recomendada uma avaliação especializada.

\section{CONCLUSÃO}

Este estudo permite uma base de comparação com a realidade internacional, atestando também em Portugal, o impacto negativo no sono da prática de adormecer a ver TV. É importante que os profissionais envolvidos na vigilância da saúde das crianças estejam conscientes desta relação, abordando este tema na sua prática clínica, com esclarecimento e reforço constante de uma boa higiene do sono, contribuindo, de uma perspetiva preventiva, para uma diminuição dos distúrbios do sono das crianças em idade escolar.

CONFLITOS DE INTERESSE: Os autores declaram a inexistência de conflitos de interesse na realização do presente trabalho.

FONTES DE FINANCIAMENTO: Não existiram fontes externas de financiamento para a realização deste artigo.

CONFIDENCIALIDADE DOS DADOS: Os autores declaram ter seguido os protocolos da sua instituição acerca da publicação dos dados de doentes.

PROTEÇÃO DE PESSOAS E ANIMAIS: Os autores declaram que os procedimentos seguidos estavam de acordo 
com os regulamentos estabelecidos pelos responsáveis da Comissão de Investigação Clínica e Ética e de acordo com a Declaração de Helsínquia da Associação Médica Mundial.

PROVENIÊNCIA E REVISÃO POR PARES: Não comissionado; revisão externa por pares.

CONFLICTS OF INTEREST: The authors have no conflicts of interest to declare.

FINANCING SUPPORT: This work has not received any contribution, grant or scholarship

CONFIDENTIALITY OF DATA: The authors declare that they have followed the protocols of their work center on the publication of data from patients.

PROTECTION OF HUMAN AND ANIMAL SUBJECTS: The authors declare that the procedures followed were in accordance with the regulations of the relevant clinical research ethics committee and with those of the Code of Ethics of the World Medical Association (Declaration of Helsinki).

PROVENANCE AND PEER REVIEW: Not commissioned; externally peer reviewed.

\section{REFERÊNCIAS}

1. Cain N., Gradisar M.Electronic media use and sleep in school-aged children and adolescents: A review. Sleep Med. 2010 ;11: 735-42. doi: 10.1016/j.sleep.2010.02.006.

2. Lelis AL, Cipriano MA, Cardoso MV, Lima FE, Araújo T. Influência do contexto familiar sobre os transtornos do sono em crianças. Rev Rene. 2014; 15:343-53.

3. Falbe J, Davison KK, Franckle RL, Ganter C, Gortmaker SL, Smith L, et al. Sleep duration, restfulness, and screens in the sleep environment. Pediatrics. 2015. 135:e367-75. doi: 10.1542/peds.2014-2306

4. Arriaga C, Brito S, Gaspar P, Luz A. Hábitos e perturbações do sono: caracterização de uma amostra pediátrica na comunidade. Acta Pediatr Port. 2015;46:367-75.

5. Silva E, Simões PA, Macedo MC, Duarte JC, Silva DM. Perceção parental sobre hábitos e qualidade do sono das crianças em idade pré-escolar. Rev Enf Ref. 2018;4: 63-72.

6. Nuutinen T, Ray C, Roos E. Do computer use, TV viewing, and the presence of the media in the bedroom predict school-aged children's sleep habits in a longitudinal study? BMC Public Health. 2013; 13: 684. doi: 10.1186/1471-2458-13-684.

7. Brockmann PE, Diaz B, Damiani F, Villarroel L, Núñez F, Bruni O. Impact of television on the quality of sleep in preschool children. Sleep Med. 2016;20:140-4. doi: 10.1016/j. sleep.2015.06.005.
8. MJ Lo. Relationship between sleep habits and nighttime sleep among healthy preschool children in Taiwan. Ann Acad Med Singapore. 2016;45:549-56.

9. Owens J, Maxim R, McGuinn M, Nobile C, Msall M, Alario A. Television-viewing Habits and Sleep Disturbance in School Children.Pediatrics. 1999;104:e27.

10. Higuchi S, Nagafuchi Y, Lee SI, Harada T. Influence of light at night on melatonin suppression in children. J Clin Endocrinol Metab. 2014;99:3298-303. doi: 10.1210/jc.2014-1629.

11. Brambilla P. Giussani M. Pasinato A, Venturelli L. Privitera F, Miraglia Del Giudice E, et al. Sleep habits and pattern in 1-14 years old children and relationship with video devices use and evening and night child activities. Ital J Pediatr. 2017; 43 : 7. doi: 10.1186/s13052-016-0324-x.

12. Calamaro CJ, Yang K, Ratcliffe S, Chasens ER. Wired at a young age: the effect of caffeine and technology on sleep duration and body mass index in school-aged children. J Pediatr Health Care. 2012;26: 276-82.

13. Cespedes EM, Gillman MW, Kleinman K, Rifas-Shiman SL, Redline S, Taveras EM. Television viewing, bedroom television, and sleep duration from infancy to mild-childhood. Pediatrics. 2014:133:e1163-71. doi: 10.1542/peds.2013-3998.

14. Silva F, Silva C, Braga L, Neto A. Hábitos e problemas do sono dos dois aos dez anos: estudo populacional. Acta Pediatr Port 2013;44:196-202.

15. Crispim J, Boto L, Melo I, Ferreira R. Padrão de sono e factores de risco para privação de sono numa população pediátrica portuguesa. Acta Pediatr Port.2011;42:93-8.

16. Lopes S, Almeida F, Jacob F, Figueiredo M, Vieira C, Carvalho F. Diz-me como dormes: hábitos e problemas de sono em crianças portuguesas em idade pré-escolar e escolar. Nascer Crescer. 2016; 25: 211-6.

17. Rangel MA Baptista C, Pitta MJ, Anjo S, Leite AL. Qualidade do sono e prevalência das perturbações do sono em crianças saudáveis em Gaia: um estudo transversal. Rev Port Med Geral Fam. 2015; 31: 256-64.

18. Silva F. Hábitos e problemas do sono das crianças dos 2 aos 10 anos. [Dissertação de candidatura ao grau de Doutor em Medicina] Lisboa: Faculdade de Ciências Médicas da Universidade Nova de Lisboa; 2014.

19. Silva FG, Silva CR, Braga LB, Neto AS. Portuguese Children's Sleep Habits Questionnaire - validation and crosscultural comparison. J Pediatr. 2014;90:78-84.

20. Parreira AF, Martins A, Ribeiro F, Silva FG. Validação Clínica da Versão Portuguesa do Questionário de Hábitos de Sono das Crianças (CSHQ-PT) em Crianças com Perturbações do Sono e PHDA. Acta Med Port. 2019:32:195-201. doi: 10.20344/ amp.10906.

21. Owens J. Classification and epidemiology of childhood sleep disorders. Prim Care. 2008; 35:533-46. 